\title{
Experimental investigation of the correlation between the dynamics of an impinging jet on a slotted plate and the acoustic field generated
}

\author{
Marwan ALKHEIR ${ }^{1, *}$, Hassan ASSOUM ${ }^{2}$, Kamel ABED-MERAÏM ${ }^{1}$, Tarek MRACH ${ }^{1}$, \\ Jana HAMDI ${ }^{1}$, Louay ELSOUFI ${ }^{3}$, Hanan $\mathrm{SKAF}^{3}$, and Anas $\mathrm{SAKOUT}^{1}$ \\ ${ }^{1}$ Laboratory of Engineering Sciences for the Environment LaSIE, Université de La Rochelle, La \\ Rochelle 17000, France. \\ ${ }^{2}$ Beirut Arab University-Tripoli Campus, Corniche El Meena, next to the Olympic Stadium, Tripoli, \\ Lebanon. \\ ${ }^{3}$ Lebanese university, faculty of sciences III, El Kobbeh, Tripoli, Lebanon.
}

\begin{abstract}
In order to know the origin of the noise generation when an impinging jet hit a specific geometry, an experimental setup was used allowing the generation of the flow and the adjustments of its parameters (such as Reynolds number, confinement, alignment, etc...). The vortex dynamics in case of a high acoustic level for two different Reynolds numbers $R e=5684$ and $R e=6214$ are considered here. Indeed, many configurations allow self-sustaining sound loop to take place in confined spaces between the nozzle and the impinged surface. This feedback loop optimizes the energy transfer between the aerodynamic field and the acoustic field and creates a source of noise that can become very noisy. Thus, to control these phenomena, it is necessary to understand the aero-acoustic coupling in such configurations. As a result, we measured the $2 \mathrm{C}$ kinematic instantaneous fields $\left(v_{x}, v_{y}\right)$ of the flow by the technique of Particle Image Velocimetry (PIV) with a sampling rate of $1 \mathrm{KHz}$ and the acoustic field is obtained using a B\&K Microphone. For the two considered Reynold numbers, we can distinguish two patterns for the vortices travelling from the nozzle toward the plate of impact.
\end{abstract}

\section{Introduction}

In certain configurations, swirling dynamics can produce loud sounds that are called whistling. This whistling occurs because of the birth and installation of a self-sustained sound loop that can sometimes be very bothersome [1]. For certain confinement ratios, impinging jets on a slotted plate optimize the energy transfer from the dynamic field to the acoustic field and gives rise to this type of whistling. Studies on this subject have been the focus of numerous studies $[2,3]$.

In this study, we measured the acoustic level for several Reynolds numbers [2]. After highlighting the acoustic peak, we used the PIV technique to understand the vortex dynamics occurring simultaneously with these peaks. Following the dynamic analysis of the flow and

by comparing the acoustic peaks with the vortex structures of the flow, one can establish the

*Marwan ALKHEIR: marwan.alkheir@univ-lr.fr 
correlations that may exist [3].

Thus, the correlation between the self-sustained sounds and the variations of the established vortex structures of flow were analyzed [1]. In this respect, an experimental device was used to generate the flows to be studied by particle image velocimetry (PIV) measurements. In this study, an $\frac{L}{H}=4$ length parameter ratio was used and Reynolds numbers $2,000 \leq R e \leq$ 12,000 were experimented.

\section{Experimental setup}

For calculating the kinematic field of the flow, PIV measurements have been carried out. The experimental device comprises a $30 \mathrm{~mJ} /$ pulse Nd YLF Pegasus New Wave laser at a frequency $1 \mathrm{KHz}$, and wavelength $\lambda=527 \mathrm{~nm}$, the frequencies of the laser pulses can go up to $20 \mathrm{KHz}$. A fast phantom $V 711$ camera with $1280 \times 800$ pixel resolution. All devices were controlled by LaVision's Davis 8.3 .0 software to calculate the kinematic field of flow. For acoustic acquisitions, we used Brüel\&Kjær 4189-A-021 microphones connected to a NI PXI-4472 dynamic acquisition board from National Instruments, and commanded by LabVIEW software.

The measurements are made for two Reynolds numbers $R e=5684$ and $R e=6214$ with a $1000 \mathrm{~Hz}$ dynamic acquisition frequency and a $5000 \mathrm{~Hz}$ acoustic acquisition frequency.

\section{Results}

Figure 1 - (a) and Figure 1 - (b), show examples of results for a length parameter ratio $\frac{L}{H}=4$. Thus, in Figure 1 - (a), we note that, passing from $R e=8006$ to $R e=8207$, the acoustic level undergoes an increase of $11.6 \%$ whereas the average evolution on the area of the Reynolds numbers studied is $0.8 \%$. Looking more closely at the swirling dynamics corresponding to a configuration of acoustic peak, we note that the deformation of the vortex structures during their passage through the slot generates the increase in the noise level. As a matter of fact, for Reynolds numbers which are responsible of creating parallel Kelvin Helmholtz structures, the large structures are deviated in the parietal flow of the plate and the acoustic levels are normal. Meanwhile, for Reynolds numbers that generate shifted structures, the latter do not approach the slot at the same time, and so they pass alternately through this slot. This passage is accompanied by deformations of these large structures and acoustic peaks. To quantify this analysis, we are interested in detecting and monitoring these vortex structures using the vorticity field, the criterion $\lambda_{2}$ and the criterion $\mathrm{Q}$ and the correlation with the acoustic signal.

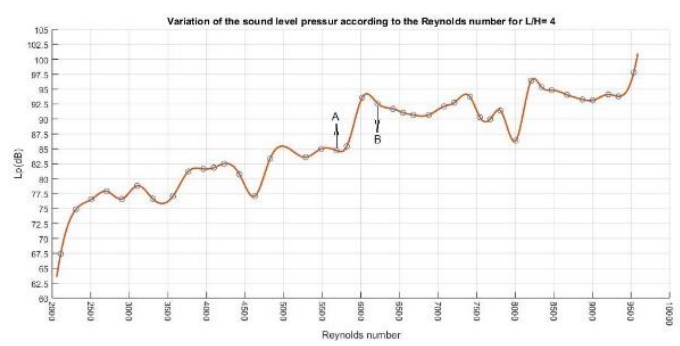

Figure 1 - (a). Evolution of the acoustic level according to Reynolds number $\left(\frac{\mathrm{L}}{\mathrm{H}}=4, \operatorname{Re}=5684\right.$ and $\left.\mathrm{Re}=6214\right)$

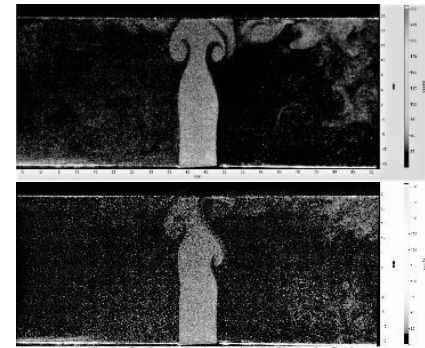

Figure 1 - (b). Vortex structures for $\mathrm{Re}=5684$ (high side) and $\mathrm{Re}=6214$ (low side). 
The vortex trajectories were detected by using the Q criterion and the $\lambda_{2}$ criterion. so we noticed the difference between the trajectories for these two Reynolds numbers. Figure 2 shows that for $\mathrm{Re}=5684$ we have only one trajectory for the vortices that pass parallel to the slot, while for $R e=6214$, two different trajectories were detected and the vortices represent a disparity, as a result a vortex passes through the slot and the second is deviated on the wall of the slotted plate. This difference in the trajectories can be the cause of the acoustic peak.

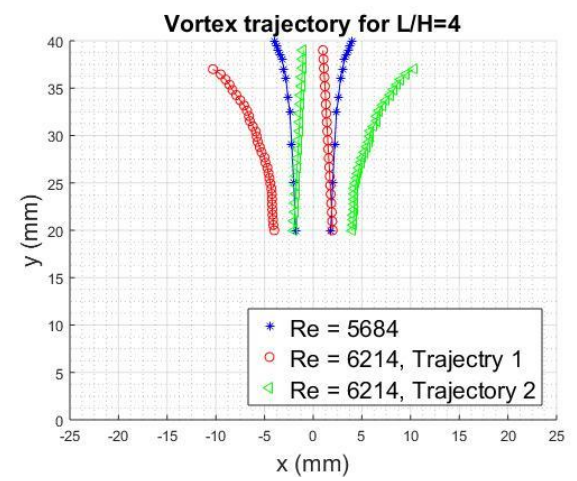

Figure 2. Trajectories of vortex structures for $\frac{\mathrm{L}}{\mathrm{H}}=4$ and for $\mathrm{Re}=5684$ and for $\mathrm{Re}=6214$.

\section{Conclusion}

Aero-acoustic coupling of a plane jet impinging on a slotted plate was considered for $\operatorname{Re}=5684, \operatorname{Re}=6214$, and for a length parameter ratio $\frac{\mathrm{L}}{\mathrm{H}}=4$. Consequently, according to the analysis of the vortex structures and their trajectories, it is shown that the two trajectories are correlated with the acoustic generations.

In perspective, in order to reduce the noise generation, we want to see the influence of the temperature on the swirling dynamics.

\section{Acknowledgments}

The authors wish to thank the region of Nouvelle Aquitaine for its financial support.

\section{References}

1. H.H. Assoum, M. El Hassan, K. Abed-Meraim, A. Sakout, The vortex dynamics and the self sustained tones in a plane jet impinging on a slotted plate, pp.231-235 Elsevier Masson, (2014).

2. H.H. Assoum, M. El Hassan, K. Abed-Meraïm, R. Martinuzzi, and A. Sakout, Experimental analysis of the aero-acoustic coupling in a plane impinging jet on a slotted plate, Fluid Dynamics, Research 45 (4), 045503, (2013).

3. H. H. Assoum, A. Sakout, K. Abed-Meraïm, A. Alia, M. El Hassan, and J. Vetel, Experimental investigation of the vibration of a slotted and a non-slotted plates and the acoustic field in a plane impinging jet, Euronoise Prague 2012, pp. 801-806 EURONOISE 2012, (2012). 